\title{
Insights on Activation Enthalpy for Non-Schmid Slip in Body Centered Cubic Metals
}

\author{
Lucas M. Hale ${ }^{\mathrm{a}, 1}$, Hojun Lim ${ }^{\mathrm{b}}$, Jonathan A. Zimmerman ${ }^{\mathrm{a}}$, Corbett C. Battaile ${ }^{\mathrm{b}}$, and Christopher \\ R. Weinberger ${ }^{\mathrm{c}}$ \\ ${ }^{a}$ Department of Mechanics of Materials, Sandia National Laboratories, Livermore, CA, 94551 \\ ${ }^{b}$ Department of Computational Materials and Data Science, Sandia National Laboratories, \\ Albuquerque, NM, 87185 \\ ${ }^{c}$ Department of Mechanical Engineering and Mechanics, Drexel University, Philadelphia, PA, \\ 19104 \\ ${ }^{1}$ Present address: Thermodynamics and Kinetics Group, National Institute of Standards and \\ Technology, Gaithersburg, MD, 20899
}

\begin{abstract}
We use insights gained from atomistic simulation to develop an activation enthalpy model for dislocation slip in body centered cubic iron. Using a classical potential that predicts dislocation core stabilities consistent with ab initio predictions, we quantify the non-Schmid stress dependent effects of slip. The kink-pair activation enthalpy is evaluated and a model is identified as a function of the general stress state. Our model enlarges the applicability of the classic Kocks activation enthalpy model to materials with non-Schmid behavior.
\end{abstract}

Keywords

Dislocations; slip; bcc metals; iron 
Plastic deformation of crystalline metals is largely controlled by dislocations: linear imperfections of the crystalline structure. Dislocation motion occurs at a critical stress associated with the resistance to slip along a particular slip system (slip plane and direction), i.e. when the thermal energy is sufficient to overcome this resistance. When only the resolved shear stress component affects slip, the material obeys Schmid's law (e.g. face-centered-cubic (fcc) metals). However, many materials, including body-centered-cubic (bcc) metals, violate this ideality [1].

At low temperatures, slip in bcc metals is dominated by thermally activated motion of a/2 $<111>$ type screw dislocations. This slip mechanism is believed to be responsible for yield that violates Schmid's Law and strong temperature and moderate strain rate sensitivities. Atomistic calculations of bcc screw dislocations have been instrumental in providing insight into the dislocation core structure and its response under stress [2-8]. This has led to the development of models that capture the dependence of slip on non-Schmid stresses [9] and that allow for implementation into dislocation dynamics $[10,11]$ and higher scale crystal plasticity [12-15] models.

Plasticity is thermally activated with approximately Arrhenius behavior relating the rate of slip, $\dot{\gamma}$, to the activation enthalpy of dislocation motion, $\Delta H$ :

$$
\dot{\gamma}=\dot{\gamma}_{0} \exp \left(\frac{-\Delta H}{k_{B} T}\right) .
$$

Here, $T$ is the temperature, $k_{B}$ Boltzman's constant, and $\dot{\gamma}_{0}$ a parameter weakly dependent on temperature. In expressing the activation enthalpy as a function of stress, the oft-used Kocks model [16] relates the enthalpy to the resolved shear stress:

$$
\Delta H=\Delta H_{0}\left(1-(\Theta)^{p}\right)^{q}
$$

where $\Delta H_{0}$ is the energy barrier under no applied stress, $p$ and $q$ are empirical constants and $\Theta$ is the ratio of the resolved shear stress to an athermal resistance to slip, i.e. $\Theta=\tau / \tau_{R}$. This stress ratio essentially enforces Schmid's law. 
In this paper, we demonstrate that the Kocks model is still applicable when Schmid's law is not obeyed, provided one appropriately chooses a stress ratio $\Theta$ dependent in the general state of stress. This choice of $\Theta$ is justified and parameterized using atomistic simulations of the kinkpair activation enthalpy associated with a screw dislocation in bcc iron. The resulting model for general stress-dependent thermally activated flow appears quite general, and may provide a starting point for other models that include non-Schmid stress-dependencies.

The initiation of slip at $0 \mathrm{~K}$ on a slip system can be written as [12]:

$$
\tau_{c r} \leq \mathbf{P}_{S}: \boldsymbol{\sigma}+\mathbf{P}_{n S}: \boldsymbol{\sigma}
$$

where $\tau_{c r}$ is a material constant, $\boldsymbol{\sigma}$ is the applied stress tensor, $\mathbf{P}_{S}$ is the Schmid projection tensor, and $\mathbf{P}_{n S}$ is a constant material dependent tensor, which we term as the non-Schmid projection tensor. Note that in this study we only consider a single slip system, but in general cases this criterion must be independently evaluated for every slip system.

The Schmid tensor is defined as

$$
\mathbf{P}_{S}=\frac{1}{2}(\mathbf{m} \otimes \mathbf{n}+\mathbf{n} \otimes \mathbf{m}) .
$$

The non-Schmid projection tensor introduces in the effects of additional stress components. Different non-Schmid tensors have been used in similar studies, and an in-depth comparison of the different models can be found in Ref. [14]. Here, we use a generalized yield model consisting of five independent projection tensors:

$$
\mathbf{P}_{n S}=c_{1} \mathbf{P}_{n S}^{\mathrm{tm}}+c_{2} \mathbf{P}_{n S}^{\mathrm{tn}}+c_{3} \mathbf{P}_{n S}^{\mathbf{n n}}+c_{4} \mathbf{P}_{n S}^{\mathrm{tt}}+c_{5} \mathbf{P}_{n S}^{\mathbf{m m}} .
$$

Here, $\mathbf{m}$ and $\mathbf{n}$ are the slip direction and slip plane normal, respectively, of the slip system, $\mathbf{t}=\mathbf{m} \times \mathbf{n}$, the $c$ 's are material constants, and the component non-Schmid tensors are represented as $\mathbf{P}_{n S}^{\mathbf{u v}}=\mathbf{u} \otimes \mathbf{v}$.

The choice of a non-Schmid dependent $\Theta$ is not obvious as a number of relationships can enforce equation (3) at $0 \mathrm{~K}$, but treat the thermal activation differently. Two examples of this are enforcing a constant denominator, i.e. $\Theta=\frac{\mathbf{P}_{s}: \mathbf{\sigma}+\mathbf{P}_{n}: \mathbf{\sigma}}{\tau_{c r}}$; or retaining the stress resolved onto the slip 
system as the numerator, i.e. $\Theta=\frac{\mathbf{P}_{s}: \boldsymbol{\sigma}}{\tau_{c r}: \mathbf{P}_{n s}: \boldsymbol{\sigma}}$. We rely on atomistic simulations to provide insight into selecting an appropriate stress ratio.

Atomistic calculations of a single $a / 2<111>$ screw dislocation are used to determine the nonSchmid constants for iron. These simulations are conducted using a previously parameterized interatomic potential for iron developed by Chamati, et al. [17] which predicts the correct equilibrium core structure, Peierls potential shape [18], and fundamental slip plane. Verifications with a second iron potential and detailed information on the two potentials are provided as supplementary material.

Systems are constructed by inserting a dislocation according to the Stroh method for anisotropic elasticity solution $[20,21]$ into a perfect bcc block of atoms with crystallographic directions and dimensions of $24 \mathrm{a}[\overline{1} 2 \overline{1}] \times 40 \mathrm{a}[\overline{101}] \times \mathrm{N}^{*} \mathrm{a}[111]$. Both thin systems, with $\mathrm{N}=4$, and thick systems, with $\mathrm{N}=48$, are considered. The z-direction is periodic, while the outer layers of atoms in the $\mathrm{x}$ - and $\mathrm{y}$-directions are held fixed. Stressing the system is accomplished through displacement control by moving the fixed boundary atoms to the strain state associated with the elasticity solution for a particular stress state. The systems are relaxed by minimizing the forces acting on the atoms that are not held fixed. This atomic configuration and a schematic of the loading orientation are shown in Figure 1.

To measure the critical stresses and activation enthalpies, stress states are applied to the system according to the following stress tensor expressed in the frame of the maximum resolved shear stress plane

$$
\sigma=\left[\begin{array}{ccc}
-P-\tau_{1} & 0 & 0 \\
0 & -P+\tau_{1}+\tau_{2} & \tau^{M R S S P} \\
0 & \tau^{M R S S P} & -P-\tau_{2}
\end{array}\right]
$$

Here, $\tau^{\text {MRSSP }}$ represents the shear stress parallel to the slip direction acting on the maximum resolved shear stress plane (MRSSP), $\tau_{1}$ and $\tau_{2}$ represent independent shear stresses perpendicular to the slip direction, and $P$ is a hydrostatic pressure. The MRSSP is at an angle $\chi$ from a $\{110\}$ type slip plane (see Figure 1). Crystalline symmetry allows for only $-30^{\circ} \leq \chi \leq$ 
$30^{\circ}$, since all other angles can be represented with respect to a different $\{110\}$ plane. To measure the critical stress, a stress state consisting of only $P, \tau_{1}$ or $\tau_{2}$ is first applied, then $\tau^{\text {MRSSP }}$ is incrementally increased until the dislocation moves. The critical resolved shear stress (CRSS) is defined as the value of $\tau^{\text {MRSSP }}$ that activates slip [3,9,15]. Thus, an alternate criterion for slip analogous to equation (3) is

$$
\tau^{M R S S P} \geq \operatorname{CRSS}(\boldsymbol{\sigma}) .
$$

Values for the non-Schmid model constants as fitted to the CRSS data are as follows: $c_{1}=0.27$, $c_{2}=0.15, c_{3}=0.31, c_{4}=-0.23, c_{5}=0.02$, and $\tau_{c r}=584 \mathrm{MPa}$. Note that the non-Schmid tensor model we use allows for effects due to both the tensile stress parallel to the dislocation line $\left(c_{5}\right)$ and the hydrostatic pressure $\left(c_{3}+c_{4}+c_{5}\right)$. There is some debate whether these components should be included in models for bcc screw dislocations [13,22,23]. For the iron potentials investigated here, we note that the tensile stress dependency is negligible; excluding its influence alters the final CRSS model values in the stress range examined $\left(\tau_{1}, \tau_{2}= \pm 114 \mathrm{MPa}\right)$ by less than half a percent. However, we do observe a slight pressure dependency which is reflected in the constants.

Nudged elastic band (NEB) calculations [24,25] are performed to calculate the activation enthalpy as a function of the entire stress state. These calculations consider end states with dislocation cores at neighboring stable positions subject to the same subcritical strain state. The activation enthalpy is measured both for the thin systems, where the dislocation is constrained to move in a homogeneous rigid fashion, and the thick systems, in which kinks translate the dislocation between Peierls valleys [26].

The activation enthalpy values from the NEB calculations are used to investigate four different expressions for $\Theta$ in equation (2). The first, denoted model A, is the classic expression

$$
\Theta=\frac{\tau}{\tau_{R}}=\frac{\mathbf{P}_{S}: \boldsymbol{\sigma}}{\tau_{R}},
$$

where $\tau_{R}$ is treated as a constant. This expression does not incorporate the non-Schmid yield criterion and is included simply for comparison. 
The other three models do satisfy the non-Schmid yield criterion and can be expressed in terms of the Schmid and non-Schmid projection tensors (equations (3-5)). Model B is taken to be the case where the denominator is held constant, as assumed in Ref. [27]. Conceptually, this represents a case where the non-Schmid stresses influence the driving force for dislocation slip, but not the resistance

$$
\Theta=\frac{\mathbf{P}_{S}: \boldsymbol{\sigma}+\mathbf{P}_{n S}: \boldsymbol{\sigma}}{\tau_{c r}} .
$$

Model $\mathrm{C}$ is for the case where the numerator remains the resolved shear stress acting on the slip system while the denominator incorporates in the non-Schmid terms. The driving force for slip remains consistent with the classic model in equation (8A), while the resistance to slip depends on the non-Schmid stresses [12]

$$
\Theta=\frac{\mathbf{P}_{S}: \boldsymbol{\sigma}}{\tau_{c r}-\mathbf{P}_{n S}: \boldsymbol{\sigma}},
$$

such that the resistance is independent of the Schmid stress. Model D is based on the slip criterion in equation (7), where the applied $\tau^{M R S S P}$ must exceed the critical value $C R S S$

$$
\Theta=\frac{\tau^{M R S S P}}{C R S S} .
$$

An expression for $C R S S$ is obtained by separating out the components of the non-Schmid model that are independent of $\tau^{M R S S P}$. For the non-Schmid model used here,

$$
C R S S=\frac{\tau_{c r}-c_{2} \mathbf{P}_{n S}^{\mathrm{tn}}: \boldsymbol{\sigma}-c_{3} \mathbf{P}_{n S}^{\mathrm{nn}}: \boldsymbol{\sigma}-c_{4} \mathbf{P}_{n S}^{\mathrm{tt}}: \boldsymbol{\sigma}-c_{5} \mathbf{P}_{n S}^{\mathrm{mm}}: \boldsymbol{\sigma}}{\cos (\chi)-c_{1} \sin (\chi)}
$$

Alternate yield models will result in different expressions for CRSS, e.g. equation (4) of Ref. [9].

In relation to the stress projections defined in equations (4) and (5), $\tau^{M R S S P}$ can be equivalently expressed in several ways:

$$
\begin{aligned}
& \tau^{M R S S P}=|\boldsymbol{\sigma} \cdot \mathbf{m} \times \mathbf{m}|, \\
& \mathbf{P}_{S}: \boldsymbol{\sigma}=\tau^{M R S S P} \cdot \cos (\chi), \\
& \mathbf{P}_{n S}^{\mathbf{m t}}: \boldsymbol{\sigma}=-\tau^{M R S S P} \cdot \sin (\chi) .
\end{aligned}
$$


Using equations 9 and 10, an equivalent expression for $\Theta$ in equation (8D) is obtained in terms of the stress projections alone

$$
\Theta=\frac{\mathbf{P}_{S}: \boldsymbol{\sigma}+c_{1} \mathbf{P}_{n S}^{\mathrm{mt}}: \boldsymbol{\sigma}}{\tau_{c r}-c_{2} \mathbf{P}_{n S}^{\mathrm{tn}}: \boldsymbol{\sigma}-c_{3} \mathbf{P}_{n S}^{\mathbf{n n}}: \boldsymbol{\sigma}-c_{4} \mathbf{P}_{n S}^{\mathrm{tt}}: \boldsymbol{\sigma}-c_{5} \mathbf{P}_{n S}^{\mathbf{m m}}: \boldsymbol{\sigma}} .
$$

Figure 2 shows the activation enthalpy curves versus the four proposed models of $\Theta$ using different stress states associated with different shear stresses perpendicular to the slip direction ( $\tau_{1}$ and $\tau_{2}$ ), different magnitudes of $\tau^{M R S S P}$, and $\chi$ angles of $\pm 30^{\circ}, \pm 15^{\circ}$ and $0^{\circ}$. All of the data shown is from the thick systems (kinked motion).

For model A, the data in Figure 2A shows increasing scatter as the Schmid stress increases. This is due to the model failing to capture the non-Schmid yield criterion. $\tau_{R}$ is arbitrarily selected to be $600 \mathrm{MPa}$ in order to show all the data points.

The remaining plots in Figure 2 show that models B, C and D successfully reproduce the nonSchmid criterion as $\Delta H$ goes to 0 as $\Theta$ goes to 1 . However, the three non-Schmid models show distinct differences for the intermediate values of $\Theta$. Out of these models, it is clear that model D exhibits the closest to universal behavior, permitting the least squares fitting procedure of equation (2) to obtain $\mathrm{p}=0.9$ and $\mathrm{q}=2.0$, with $\Delta H_{0}=0.6 \mathrm{eV}$ (solid line in Figure 2D). Thus equation (8D) (or equation (11)) can be used to accurately model the activation enthalpy at various stress states for bcc metals with non-Schmid dependencies. The only noticeable limitation is that this model is unable to capture the slight variations in $\Delta H$ observed at $\tau^{M R S S P}=0$ for different applied perpendicular shears.

Performing the same analysis using the thin systems to enforce rigid slip reveals similar results in that model D collapses the data. However, fitting the rigid slip data to equation (2) obtains different values for $p$ and $q$ (1.1 and 2.5 respectively). The dashed line in Figure 2D is representative of this model. While here the two resulting Kocks models are very similar our investigations with another iron potential reveal this to not be universal (see supplementary materials). 
These results demonstrate that model D collapses the data into a single line that allows for the activation energy (equation (2)) to be generalized to cases where thermally activation is dependent on stresses not resolved onto the slip plane. However, equation (11) appears specific to yield in BCC metals. Nonetheless, the form can be generalized by decomposing $\Theta$ into two parts: the numerator that depends on the stress resolved into the Burgers vector; and the denominator which does not depend on this stress. This suggests that the stress should be decomposed into components $\tau^{M R S S P}$ and $\mathbf{s}$, where $\mathbf{s}$ is the resultant stress tensor that does not depend on $\tau^{\text {MRSSP }}$, such that for thermal activation, $\Theta$ should be:

$$
\Theta=\frac{f\left(\tau^{M R S P}\right)}{g(\mathbf{s})} .
$$

This is a general model that clearly captures the limiting case of Schmid behavior, where $g(\mathbf{s})$ is a constant and the function $f()$ the resolved shear stress, recovering the notion of thermally activated flow.

Conceptually, this model is consistent with classic dislocation theory and experimentally reported slip behavior in bcc metals. $\tau^{\text {MRSSP }}$ is equivalent to the driving force acting on a screw dislocation in an isotropic medium given by the Peach-Koehler equation. With the driving force on a screw dislocation independent of the slip plane, the crystallographic information manifests only in the lattice resistance. At low temperatures, only certain motions (associated with highly packed crystallographic planes) offer low resistance to the dislocation motion to produce crystallographic slip. At higher temperatures, the thermal energy allows for the dislocation to overcome the resistance for alternate directions of motion, leading to cross slip. At high enough temperatures, thermal energy can lead to negligible lattice resistance such that dislocation motion only is defined by the driving force, e.g. pencil glide.

The results presented here allow for the development of bcc plasticity models which capture accurate non-Schmid and temperature dependent slip behaviors [29]. These results also provide insight into general models of thermally activated processes in plasticity where the resistance to slip is dependent on stress components other than the resolved shear stress. However, further verification on other systems is necessary to demonstrate generality. 


\section{Acknowledgements}

This work is supported by the Advanced Simulation and Computing - Physics and Engineering Models (ASC-P\&EM) program at Sandia National Laboratories. Sandia is a multi-program laboratory managed and operated by Sandia Corporation, a wholly owned subsidiary of Lockheed-Martin Corporation, for the United States Department of Energy's National Nuclear Security Administration under contract DE-AC04-94AL85000. 
References

[1] J.W. Christian, Met. Trans. 14A (1983) 1237-1256.

[2] M.S. Duesbery, V. Vitek, Acta Mater. 46 (1998) 1481-1492.

[3] R.Groger, A.G. Bailey, V. Vitek, Acta Mater. 56 (2008) 5401-5411.

[4] V. Vitek, Proc. Roy. Soc. Lond. A 352 (1976) 109-124.

[5] S. Takeuchi, E. Kuramoto, Scripta Metall. 8 (1974) 785-789.

[6] C.R. Weinberger, C.C. Battaile, B.L. Boyce, Int. Mater. Rev. 58 (2013) 296-314.

[7] M. Wen, A.H.W. Ngan, Acta Mater. 48 (2000) 4255-4265.

[8] V. Vitek, M. Mrovec, J.L. Bassani, Mat. Sci. Eng. A 365 (2004) 31-37.

[9] R. Groger, V. Racherla, J.L. Bassani, V. Vitek, Acta Mater. 56 (2008) 5412-5425.

[10] Z. Wang, I. Beyerlein, Int. J. Plasticity 27 (2011) 1471-1484.

[11] K. Srivastava, R. Gröger, D. Weygand, P. Gumbsch, Int. J. Plasticity 47 (2013) 126-142.

[12] C.R. Weinberger, C.C. Battaile, T.E. Buchheit, E.A. Holm, Int. J. Plasticity 37 (2012) 1630.

[13] A. Koester, A. Ma, A. Hartmaier, Acta Mater. 60 (2012) 3894-3901.

[14] H. Lim, C.R. Weinberger, C.C. Battaile, T.E. Buchheit, Modelling Simul. Mater. Sci. Eng. 21 (2013) 045015.

[15] R. Groger, V. Vitek, Acta Mater. 56 (2008) 5426-5439.

[16] U.F. Kocks, Prog. Mater. Sci. 19 (1975) 110-170.

[17] H. Chamati, N.I. Papanicolaou, Y. Mishin, D.A. Papaconstantopoulos, Surface Science 600 (2006) 1793-1803.

[18] L. Ventelon, F. Williaime, J. Computer-Aided Mater. Des. 14 (2007) 85-94.

[19] L. Proville, D. Rodney, M.-C. Marinica, Nat. Mater. 11 (2012) 845-849.

[20] A.N. Stroh, Philos. Mag. 3 (1958) 625-646.

[21] A.N. Stroh, J. Math. Phys. 41 (1962) 77-103.

[22] M.S. Duesbery, Proc. Roy. Soc. Lond. A 392 (1984) 145-173.

[23] R. Groger, Philos. Mag. 94 (2014) 2021-2030.

[24] H. Jonsson, K. Mills, K.W. Jacobsen, in: B.J. Berne, G. Ciccotti , D.F. Coker (Eds.), Classical and Quantum Dynamics in Condensed Phase Simulations, World Scientific, Singapore, 1998, pp. 385-404.

[25] G. Henkelman, B.P. Uberuaga, H. Jonsson, J. Chem. Phys. 113 (2000) 9901-9904. 
[26] P.A. Gordon, T. Neeraj, Y. Li, J. Li, Modelling Simul. Mater. Sci. Eng. 18 (2010) 085008.

[27] T.E. Buchheit, C.C. Battaile, C.R. Weinberger, E.A. Holm, JOM 63 (2011) 33-36.

[28] M.R. Fellinger, H. Park, J.W. Wilkins, Phys. Rev. B 81 (2010) 144119.

[29] H. Lim, L.M. Hale, J.A. Zimmerman, C.C. Battaile, C.R. Weinberger, Int. J. Plasticity (Accepted). 
Figure 1: (A) Schematic showing the orientation of the applied stress, the slip plane and the MRSSP. Adapted from Ref. [28]. (B) Image of the atomic configuration outlining the fixed and relaxed regions of atoms, and the range of MRSSP investigated.

Figure 2: Activation enthalpy of kinked dislocation slip versus different stress normalizations. 

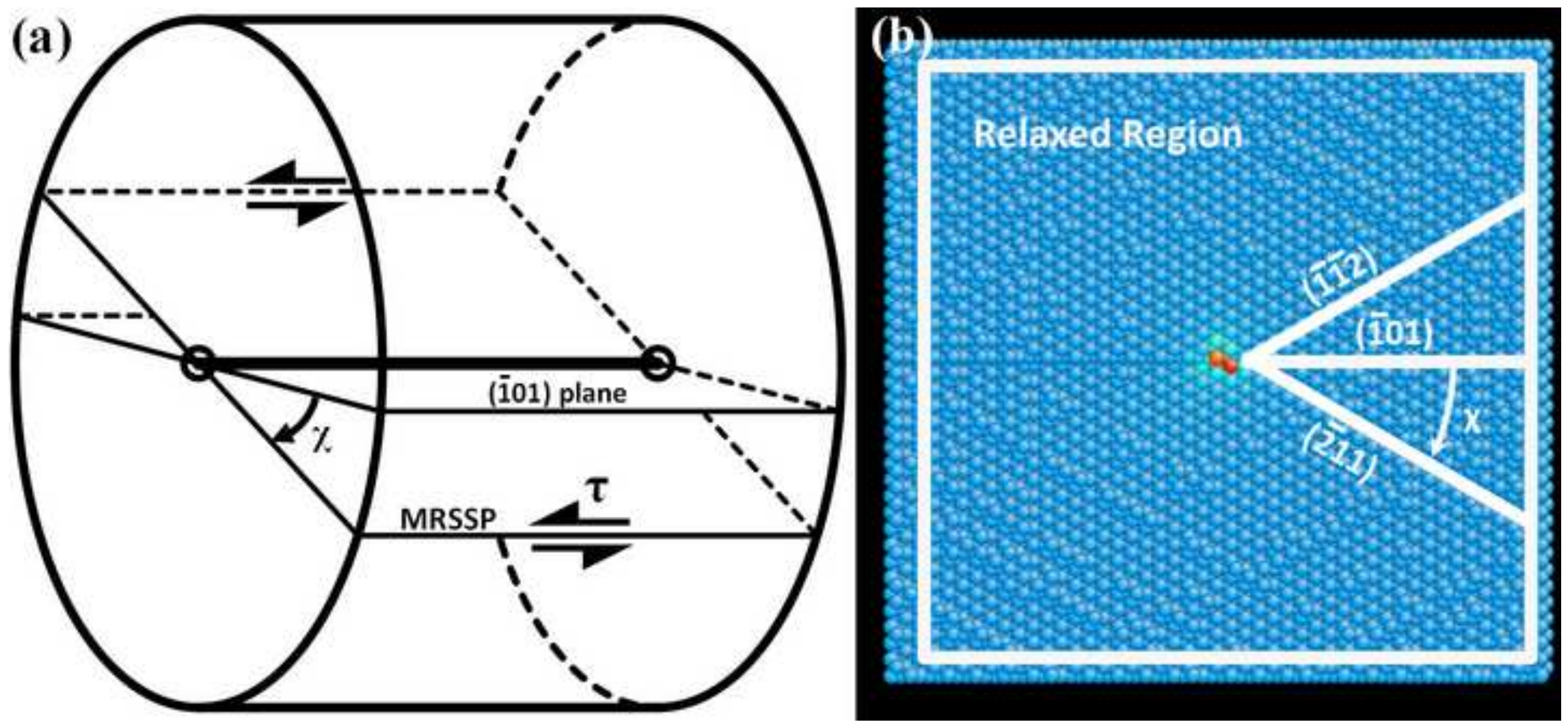

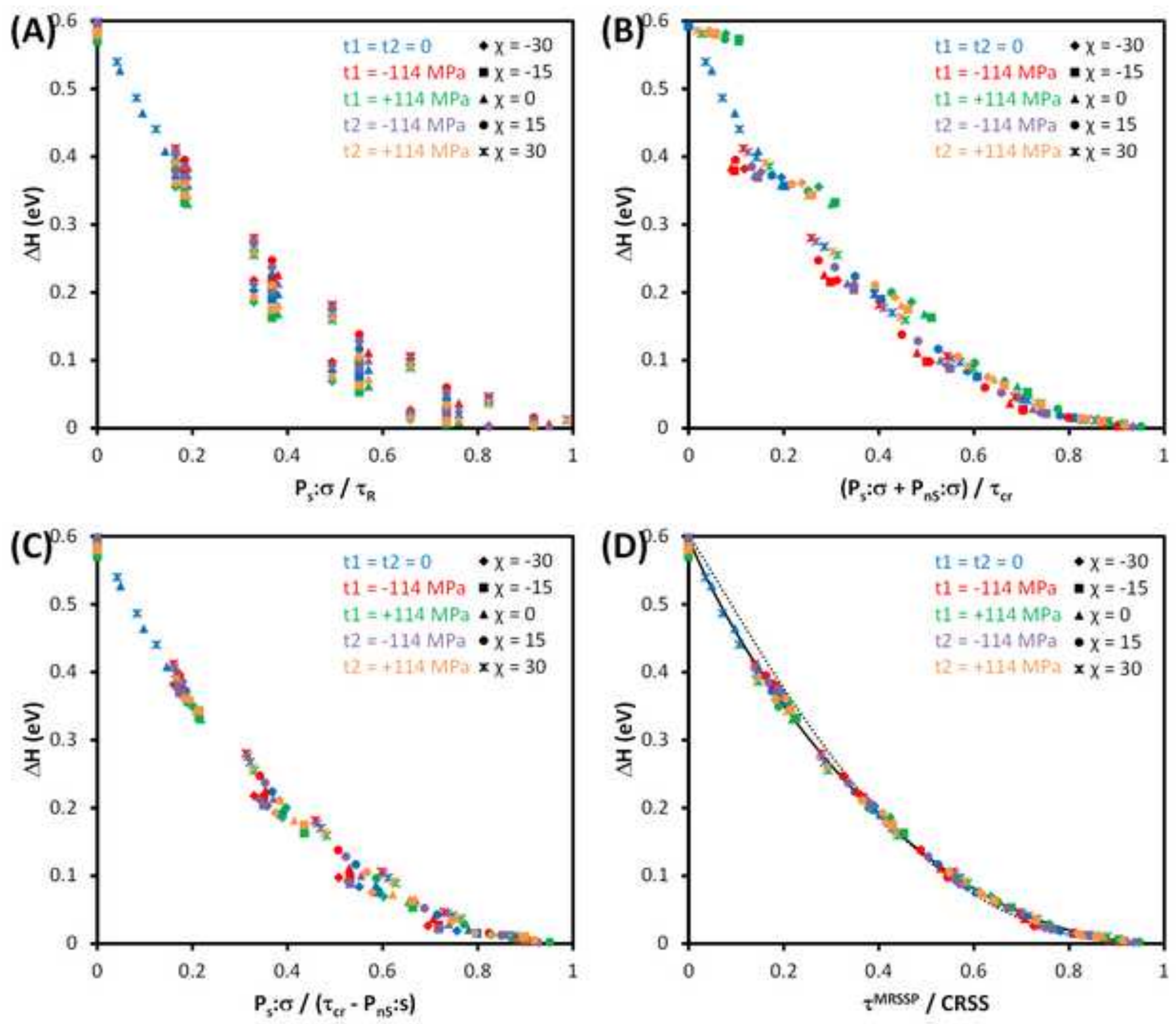